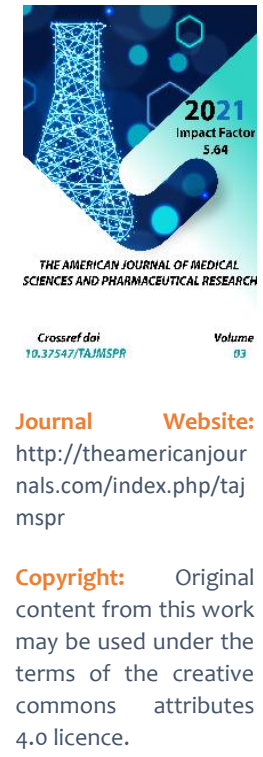

\title{
The Meaning Of Questionnaires In Assessing The Quality Of Life Of Patients With Type 2 Diabetes On Software Hemodialysis
}

\author{
Kholikov Alisher Yusupovich \\ Head Of The Department Of Hemodialysis, Republican Specialized Scientific And Practical \\ Medical Center For Endocrinology Named By Acad. Ya.Kh. Turakulova, 100125, Tashkent, \\ Republic Of Uzbekistan
}

\author{
Urmanova Yulduz Makhkamovna \\ Doctor Of Medical Sciences, Associate Professor, Tashkent Pediatric Medical Institute, \\ Tashkent, Republic Of Uzbekistan
}

\section{ABSTRACT}

The aim of the study. To study the clinical characteristic and quality of life of the patients with type 2 diabetes mellitus.

Material and methods. We examined and examined a total of 80 patients with diabetic nephropathy on program hemodialysis for the period from January 1, 2018, to January 1, 2020.

Results. In this article, the authors analyze 80 cases of diabetic nephropathy that were on programmed hemodialysis. Of these, 32 women, 48 men. The results also confirm the literature that patients with type 2 diabetes mellitus have a low quality of life indicators before hemodialysis.

Conclusions. A questionnaire to determine the quality indicators - WHOQOL-BREF, MMSE, Hamilton depression scale are the most sensitive and informative to determine the quality of life in patients with chronic kidney disease.

\section{KEYWORDS}

Diabetes mellitus type 2, chronic kidney disease, quality of life

\section{INTRODUCTION}

As is known, chronic kidney disease (CKD), characterized by a decrease in the excretory and creator function of the kidney, leads to a violation of the homeostasis of the inner environment of the body. In case of kidney diseases, accompanied by arterial 
hypertension, disorders of protein, lipid metabolism, water-salt and acid-base homeostasis, conditions for pathogenic effect on It is known that it is in kidney diseases that are accompanied by arterial hypertension, disorders of protein, lipid metabolism, watersalt and acid-base homeostasis, there are conditions for pathogenic influence on the nervous system. Therefore, the problem of CKD, especially in its terminal stage, in recent years, becomes more and more medical and social importance. The syndromes of the damage to the nervous system at the CKD are known for a long time. Thus, uremic polyneuropathy was first described in the XIX century. Since then, there have been many publications on this topic. Nevertheless, issues relating to the prevalence and clinical options for the defeat of the central and peripheral nervous system in patients with CKD studied little, and methods for the prevention and treatment of neurological complications in this category of patients are not developed sufficiently. Thus, according to the authors, uremic polyneuropathy has the character of asymmetric, distal, sensing neuropathy with a preferable lesion of the lower extremities [610]. This condition occurs in $50-60 \%$ of patients with terminal renal failure, significantly worsening the quality of life (QoL) of patients $[9,12,14]$. At the same time, the patterns of pathogenesis and sangenesis, rational and early diagnosis of neuropathy in patients in chronic hemodialysis, are not studied [16-18].

At the present stage, the issue of improving the QoL of this category of patients becomes more and more relevant to the introduction of new more advanced dialysis technologies, kidney transplantation and methods of healthcare correction. All over the world, interest in the use of common tools for early detection of cerebrovascular complications by assessing QoL with a wide range of diseases and various states is increasing. Simple and affordable research tools are attracted special attention, including the WHOQOL-BREF questionnaire. The WHOQOL-BREF brief questionnaire consists of 26 points that evaluate the following wide areas: physical health, psychological health, social relations and the environment. The WHOQOL-BREF brief questionnaire is a reduced version of the original technique.

MINI-MENTAL STATE EXAMINATION (MMSE) test. A brief scale of mental status assessment (eng. Mini-Mental State Examination, MMSE) is a short questionnaire from 30 points, widely used for primary assessment of the condition of cognitive functions and screening their violations, including dementia.

HAMILTON DEPRESSION SCALE. The Hamilton scale to assess depression is among the most famous, reliable recognized by experts. The scale is well balanced: its sensitivity according to the results of meta-analysis 24 of serious studies is $84 \%$, and specificity is $83 \%$. All of the above questionnaires were used by us during the study and repeated every six months in the dynamics of treatment. The main purpose of the following study made in 2019 was to assess the equivalence of the measurements of the English, Chinese and Malay versions of WHOQOL-BREF. The authors analyzed the data from the previously published crossstudy research of Wonders and used linear regression models to adjust the possible interfering variables. It was established that the version of the WHOQOL-BREF questionnaire in English, Chinese and Malays can be considered equivalent to the evidence in the same areas more reliable than in others. Given the large number of people speaking/reading in Chinese and Malay, this study is widely relevant $[19,20]$.

Italian authors in 2017 were conducted and published a systematic meta-analysis to determine whether hemodialysis or peritoneal 
dialysis ensures a better quality of life. The authors were searching in the Cinahl, MEDLINE, PubMed, Scopus and Proquest database, including articles published from 2011 to June 2016. They selected articles in which, with the help of KDQOL-SF 1.3 or 36 questionnaires, they compared the quality of the life of patients on hemodialysis and peritoneal dialysis. The data was collected using Excel Office, and for independent samples, a T-test was conducted to identify significant differences. Results: Only some of the seven articles found significant differences between the two methods of treatment [12].

All of the above emphasizes the relevance of this direction. In this regard, we formulated the following purpose of research work. The purpose of the study is to assess the quality of the life of patients with SD 2 type before treatment with hemodialysis using the WHO brief questionnaire to assess the quality of life (WHOQOL-BREF), a brief scale of estimating the cognitive function MMSE and the Hamilton depression scales, taking into account the degree of chronic brain ischemia.

\section{MATERIALS AND METHODS}

We examined and examined 80 patients suffering from type 2 diabetes, with CPR V Art. on program hemodialysis. Of these, women were-32, men -48 . The average age of men amounted to $67 \pm 4.2$ years, and the average age of women is $64 \pm 5.6$ years. 20 patients of the relevant age amounted to a group of control. The number of hemodialysis sessions in patients varied from 2 to 162 .

Inclusion criteria: patients with type 2 diabetes mellitus, located on software hemodialysis, with HBP III Art., Diabetic nephropathy 5 tbsp.

Exception Criteria: Pregnant women, children and young people with type 1 SD 1, patients with cardiovascular pathology before establishing the diagnosis of type 2 SD 2, autoimmune thyroiditis (hypothyroidism). According to the degree of chronic brain ischemia (HIBM), patients have divided into 3 groups: 1 gr. - 30 (37.5\%) patients with diabetic nephropathy 5 stages with HIBM 1 degree; 2 gr. - 26 (32.5\%) patients with diabetic nephropathy 5 stages with HIBM 2 degrees; 3 gr. - 24 (30\%) patients with diabetic nephropathy 5 stages with HIBM 3 degrees. All 80 patients were performed by all studies that included generally clinical (general blood test, general urine analysis and non-Correne), biochemical (blood sugar, glycemic profile, glycated haemoglobin $\mathrm{HBA}_{1} \mathrm{C}$, urea, creatinine, blood electrolytes, lipid spectrum, correlogram, etc.), Hormonal blood tests, BDNF, S100, NSE in the blood, ECG, ultrasound of the internal organs, dopplerography of the main arteries of the head, if necessary, patients were sent to the increase in the radiological, ultrasound of internal and genital organs, consultation of the cardiologist, neurologist, nephrologist, oculist, surgeon, and Dr Research. The quality of life of the patients we were assessed to hemodialysis and in the dynamics of treatment with the WHO brief questionnaire to assess the quality of life (WHOQOL-BREF) and the depression scales of Hamilton. The WHOQOL-BREF brief questionnaire consists of 26 points, which evaluate the following 4 wide areas: physical health, psychological health, social relations and the environment. The WHOQOL-BREF brief questionnaire is a reduced version of the original technique. A brief scale of assessment of cognitive functions or Mini-Mental State Examination (MMSE) A short questionnaire out of 30 points, widely used for the primary assessment of the condition of cognitive functions and screening their violations, including dementia. Any rating that is more than 27 (out of 30) is effective normal. Below this, 20 - 26 indicates a slight dementary; 10 19 moderate dementia, and below 10 - severe dementia. The total score of the first 17 points 
of the Hamilton scale, which determines the severity of the depressive disorder, has the following interpretation: 0-7 Balls - norm, 8-13 B- Easy depressive disorder, 14-18B depressive medium degree disorder 19-22b depressive disorder severe, more than $23 \mathrm{~b}-\mathrm{a}$ depressive disorder of extremely severe severity. The obtained data were processed using Microsoft Excel and Statistica_6 computer programs. The average arithmetic (m) was calculated, the standard deviation of the medium-average or an average arithmetic error of all $\mathrm{N}$ repeats $(M)$. The presence of differences in the level between groups was estimated by the value of the confidence interval and the Student's criterion ( $P$ ). Differences were considered statistically significant at $p<0.05$.

\section{RESEARCH RESULTS}

Table 1 shows the distribution of inspected patients by sex and age. As can be seen from Table 1, patients prevailed in the age category from 60 to 74 years as among men and women - $18 / 20$ cases, respectively.

Table 1. Distribution of patients by gender and age (WHO, 2017)

\begin{tabular}{|c|c|c|}
\hline Age, years & Number of men & Number of women \\
\hline $18-44$ (young age) & $7(14,5 \%)$ & $6(18,7 \%)$ \\
\hline $45-59$ (middle-aged) & $13(27,0 \%)$ & $6(18,7 \%)$ \\
\hline $60-74$ (elderly age) & $18(37,5 \%)$ & $20(62,5 \%)$ \\
\hline $\begin{array}{c}75 \text { and older } \\
\text { (senile age) }\end{array}$ & $10(20,8 \%)$ & - \\
\hline Total: $\mathbf{n}=80$ & $48(60 \%)$ & $32(40 \%)$ \\
\hline
\end{tabular}

Patients during inspection presented various complaints that indicated the presence of Him. Most often met such complaints as fast fatigue - 80 (100\%), headaches - 78 patients (97.5\%), dizziness - $63(78.7 \%)$, reduction of memory - 52 (65\%), disruption of sleep formula - 48 (60\%)\%, pain in the heart - 37 (46.3)\%, that is, these complaints are most characteristic of chronic vascular insufficiency and were dominant. The next stage of the work was to study the quality of life of patients using a brief Questionnaire WHO for assessing the quality of life (WHOQOL-BREF) and the depression scales of Hamilton in Table 2 and 3, the frequency of identified violations among 80 patients was given with the help of these studies. 
Table 2. WHO brief questionnaire results for life quality assessment (WHOQOL-BREF) in groups

\begin{tabular}{|c|c|c|c|c|c|c|c|c|}
\hline \multirow{2}{*}{ № } & \multirow{2}{*}{ Indicators } & \multicolumn{2}{|c|}{$\begin{array}{c}\text { Control } \\
\text { Group }\end{array}$} & \multicolumn{2}{|c|}{ patients in the group } & \multicolumn{2}{c|}{$\begin{array}{c}\text { \% to the total number of } \\
\text { patients in the group }\end{array}$} \\
\cline { 5 - 9 } & $\begin{array}{c}1 \mathrm{gr} \\
\mathrm{n}=30\end{array}$ & $\begin{array}{c}2 \mathrm{gr} \\
\mathrm{n}=26\end{array}$ & $\begin{array}{c}3 \mathrm{gr} \\
\mathrm{n}=24\end{array}$ & $\begin{array}{c}1 \mathrm{gr} \\
\mathrm{n}=30\end{array}$ & $\begin{array}{c}2 \mathrm{gr} \\
\mathrm{n}=26\end{array}$ & $\begin{array}{c}3 \mathrm{gr} \\
\mathrm{n}=24\end{array}$ \\
\hline & $\begin{array}{c}\text { Physical and } \\
\text { psychological } \\
\text { Welfare }\end{array}$ & $50 \pm 7,6$ & $19 \pm 2,3^{*}$ & $13 \pm 3,5^{*}$ & $12 \pm 1,2^{*}$ & $29 \%$ & $34 \%$ & $37 \%$ \\
\hline 2 & $\begin{array}{c}\text { Self- } \\
\text { perception }\end{array}$ & $46 \pm 4,2$ & $22 \pm 3,2^{*}$ & $14 \pm 1,2^{*}$ & $10 \pm 0,5^{*}$ & $40 \%$ & $13 \%$ & $47 \%$ \\
\hline 3 & $\begin{array}{c}\text { Microsocial } \\
\text { support }\end{array}$ & $39 \pm 0,6$ & $11 \pm 0,6^{*}$ & $8 \pm 0,7^{*}$ & $4 \pm 0,2^{*}$ & $60 \%$ & $33 \%$ & $7 \%$ \\
\hline 4 & $\begin{array}{c}\text { Social well- } \\
\text { being }\end{array}$ & $58 \pm 5,7$ & $33 \pm 5,7^{*}$ & $34 \pm 3,9^{*}$ & $15 \pm 0,2^{*}$ & $63 \%$ & $19 \%$ & $18 \%$ \\
\hline
\end{tabular}

Note: * - the accuracy of differences with control, where * $-p<0.05$

As can be seen from Table 2, in all groups of patients prior to hemodialysis, indicators of the quality of life by WHOQOL-BREF questionnaire compared with the control group ( $p$ <0.05). At the same time, the "physical and psychological well-being" component was lower in patients with 3 groups, the "self-perception" component was significantly lower in patients with 3 groups ( $p$ $<0.05)$. The same trend was detected relative to the "Micro-Provision Support" components and "Social well-being ", Which were also reliably be low in patients with 3 groups.

Table 3. Results of Hamilton Depression scale by Groups

\begin{tabular}{|c|c|c|c|c|c|c|c|c|}
\hline \multirow{2}{*}{ № } & \multirow{2}{*}{ Disorders } & \multirow{2}{*}{$\begin{array}{l}\text { Control } \\
\text { group }\end{array}$} & \multicolumn{3}{|c|}{ patients in the group } & \multicolumn{3}{|c|}{$\begin{array}{l}\% \text { to the total number of patients } \\
\text { in the group }\end{array}$} \\
\hline & & & $\begin{array}{l}1 \mathrm{gr} \\
\mathrm{n}= \\
30\end{array}$ & $\begin{array}{c}2 g r \\
n=26\end{array}$ & $\begin{array}{c}3 g r \\
n=24\end{array}$ & $\begin{array}{c}1 \mathrm{gr} \\
\mathrm{n}=30\end{array}$ & $\begin{array}{c}2 g r \\
n=26\end{array}$ & $\begin{array}{c}3 g r \\
n=24\end{array}$ \\
\hline 1 & $\begin{array}{c}\text { Easy } \\
\text { depressive } \\
\text { disorder }\end{array}$ & \multirow[b]{2}{*}{$5,4 \pm 0,3$} & $\begin{array}{l}9,5 \pm \\
0,6 *\end{array}$ & $\begin{array}{c}11,5 \pm 0,4 \\
*\end{array}$ & $\begin{array}{c}13,2 \pm 0,3^{*} \\
*\end{array}$ & $42 \%$ & $19 \%$ & $39 \%$ \\
\hline 2 & $\begin{array}{c}\text { Depressive } \\
\text { Middle } \\
\text { Severity } \\
\text { Disorder }\end{array}$ & & $\begin{array}{l}15,7 \pm \\
0,2 *\end{array}$ & $\begin{array}{c}17,2 \pm 0,9 \\
*\end{array}$ & $\begin{array}{c}18,4 \pm 0,6 * \\
*\end{array}$ & $41 \%$ & $17 \%$ & $42 \%$ \\
\hline
\end{tabular}




\begin{tabular}{|c|c|c|c|c|c|c|c|c|}
\hline 3 & $\begin{array}{c}\text { depressive } \\
\text { disorder of } \\
\text { severe more }\end{array}$ & & $\begin{array}{c}19,2 \pm \\
0,7^{*}\end{array}$ & $\begin{array}{c}18,5 \pm 0,3 \\
*\end{array}$ & $\begin{array}{c}20,2 \pm 0,4^{*} \\
*\end{array}$ & $7 \%$ & $37 \%$ & $56 \%$ \\
\hline
\end{tabular}

Note: * - the accuracy of differences with control, where * $-p<0.05, * *-p<0.01$

As can be seen from Table 3, in patients of $3 x$ groups discovered various impaired emotional spheres: a slight depressive disorder dominated in patients with $1 \mathrm{gr}-42 \%$, the depressive disorder of the average severity was established equally in 1 and 3 groups (41\% and $42 \%$ Accordingly), the depressive disorder of severe more often met in patients with $3 \mathrm{gr}$.
- 56\%. In addition, the accuracy of the differences in the Hamilton scale indicators compared with the control in all groups of patients $(P<0.05)$ and to a greater extent of 3 groups. ( $p<0.01)$. Table 4 shows the average values of the points used in questionnaires in patients with SD 2 in groups.

Table 4. Medium values of the points used questionnaires in patients depending on the degree of chronic brain ischemia

\begin{tabular}{|c|c|c|c|c|c|c|c|}
\hline \multirow{2}{*}{$g r$} & \multirow{2}{*}{ Total } & \multicolumn{4}{|c|}{ WHOQOL-BREF } & \multirow{2}{*}{$\begin{array}{l}\text { Test of } \\
\text { MMSE }\end{array}$} & \multirow{2}{*}{$\begin{array}{l}\text { Hamilton } \\
\text { Depression } \\
\text { scale }\end{array}$} \\
\hline & & 1 & 2 & 3 & 4 & & \\
\hline 1 & $n=30$ & $19 \pm 2,3^{*}$ & $22 \pm 3,2$ & $11 \pm 0,6$ & $33 \pm 5,7^{*}$ & $23 \pm 1,4$ & $11 \pm 0,2$ \\
\hline 2 & $n=26$ & $13 \pm 3,5^{*}$ & $14 \pm 1,2$ * & $8 \pm 0,7^{*}$ & $34 \pm 3,9 *$ & $19 \pm 1,7^{*}$ & $18 \pm 1,4^{*}$ \\
\hline 3 & $n=24$ & $12 \pm 1,2^{*}$ & $10 \pm 0,5^{*}$ & $4 \pm 0,2 *$ & $15 \pm 0,2 *$ & $9 \pm 1,2^{*}$ & $25 \pm 1,6^{*}$ \\
\hline \multicolumn{8}{|c|}{ Control } \\
\hline 2 & $n=20$ & $50 \pm 7,6$ & $46 \pm 4,2$ & $39 \pm 0,6$ & $58 \pm 5,7$ & $30 \pm 1,2$ & $3 \pm 0,4$ \\
\hline
\end{tabular}

Note: * - P - Statistical significance, 1 - Physical and psychological well-being, 2 - self-perception, 3 Micro-social support, 4 - Social well-being

Table 4 shows that patients with SD 2 depending on the degree of Him data on 3 questionnaires were significantly different from the control group (healthy persons) in the estimation of the 4xqol-Bref, MMSE test and the Hamilton depression scales. This indicated a decrease in criticism of his own health state and the presence of reduced selfesteem on the WHOQOL-BREF questionnaire.
In determining the degree of depression on the Hamilton scale, it was revealed that in these patients as the degree of depression increases, and the degree of depression increased. As for the MMSE test, its results showed the presence of a decrease in cognitive functions at the level of lung dementia in the 1 group and severe dementia in the group of patients. Thus, the study of the 
quality of life of patients using a brief WHO Questionnaire to assess the quality of life (WHOQOL-BREF) and the Hamilton Depression scales in 3-type 3 patients with diabetes mellitus prior to conducting program hemodialysis revealed a significant lag behind the above indicators of the quality of life in all groups of patients. These statistical data indicate the need for further research in the dynamics of program hemodialysis. So, from the survey among 80 patients, we identified various violations of the Qol and the emotional sphere. Thus, in all groups of patients prior to hemodialysis, indicators of the quality of life on the WHOQOL-BREF questionnaire compared with the control group ( $p$ <0.05). At the same time, the "physical and psychological well-being" component was lower in patients with 3 groups, the "self-perception" component was significantly lower in patients with 3 groups ( $p$ $<0.05$ ). The same trend was detected relative to the "Micro-Provision Support" components and "Social well-being ", which were also reliably low in patients with 3 groups. The indicators of the Hamilton scale revealed disorders from a light depressive disorder, which is most often detected in patients $1 \mathrm{gr}$ $42 \%$, to the depressive disorder of severe, more frequently occurring in patients 3 gr. $56 \%$ Indicators of the quality of life in patients with HBP under conservative therapy and dialysis below, and the level of anxiety and depression on the Hamilton scale is higher than the corresponding indicators of healthy individuals. The results also confirm the literature data that patients with type 2 diabetes prior to program hemodialysis have low-quality indicators.

\section{CONCLUSIONS}

1) A questionnaire to determine the quality indicators - WHOQOL-BREF, MMSE, Hamilton depression scale are the most sensitive and informative to determine the quality of life in patients with CKD.

2) Assessment of quality of life using the WHOQOL-BREF scale, determining the level of anxiety and depression on the Hamilton scale, as well as the assessment of cognitive functions for a brief scale MMSE must act as the criterion of clinical and functional severity of the state and the effectiveness of the treatment of therapy in patients with CKD in conditions therapy by various methods.

\section{REFERENCES}

1. Vasilyeva, I. A. (2005). Quality of life of patients on chronic hemodialysis. Nephrology. T. 9, No. 3. pp. 48-54.

2. Vasilyeva, I. A. (2008). Psychological adaptation to the disease in individuals with chronic renal failure. Reviews of psychiatry and medical psychology, .-pp. 27-30.

3. Vasilyeva, I. A. (2010). Quality of life of patients with hemodialysis treatment: biological and psychosocial factors, assessment methods and approaches to correction: author. dis. ... Dr Psychol. science - St. Petersburg.,. p.45.

4. Gendlin G. E. (2005). Study of the quality of life of patients receiving renal treatment with hemodialysis and after 
ATP with the help of universal questionnaire SF-36. Nephrology and dialysis, T. 7, No. 3. P. 284.

5. Gorin, A.A. (2005). Quality of life and border mental disorders in patients on program hemodialysis: author. dis. ... Cand. honey. science -M., - 24 p.

6. Krylova, M. I. (2011). Quality of life of patients on hemodialysis and peritoneal dialysis: author. dis. ... Cand. honey. science - M., - 26

7. Tiller, L. M. (2003). Quality of life and the functional state of the vegetative nervous system in patients with chronic renal failure: author. dis. ... Cand. honey. science - Tomsk, - $25 \mathrm{p}$.

8. Capa J.P., Abdelmalac B, Farag E. (2011). Neurological biomarkers in the perioperative period. British Journal of Anesthesia. V107, N6. P. 844-85813.

9. Tomaszewski, D. (2015). Biomarkers of brain damage and postoperative cognitive disorders in orthopaedic patients: an update. BioMed research international, 2015.

10. Donato, R., Riuzzi, F., \& Sorci, G. (2013). Causes of elevated serum levels of S100B protein in athletes. European journal of applied physiology, 113(3), 819-820.

11. Elif D.B., Mustaf A., Serdar K. (2013). Comparison of the effect of desflurane and propofol anaesthesia on the inflammatory response and S100 $\beta$ protein during coronary artery bypass grafting. Inflammatory, V. 36, N6. P. 1327-1333.

12. Jones, E. L., Gauge, N., Nilsen, O. B., Lowery, D., Wesnes, K., Katsaiti, E., ... \& Ballard, C. (2012). Analysis of neuronspecific enolase and $\mathrm{S} 100 \mathrm{~B}$ as biomarkers of cognitive decline following surgery in older people. Dementia and geriatric cognitive disorders, 34(5-6), 307-311.

13. Zazzeroni, L., Pasquinelli, G., Nanni, E., Cremonini, V., \& Rubbi, I. (2017). Comparison of quality of life in patients undergoing hemodialysis and peritoneal dialysis: a systematic review and metaanalysis. Kidney and Blood Pressure Research, 42(4), 717-727.

14. Ozer, E., \& Yilmaz, R. (2017). Effect different anesthetic techniques on mental outcome in elderly patients undergoing off-pump coronary artery bypass graft surgery. J. cardiovasc. Science, 29(1), 17-22.

15. Salama, I., Malone, P. S., Mihaimeed, F., \& Jones, J. L. (2008). A review of the $S 100$ proteins in cancer. European Journal of Surgical Oncology (EJSO), 34(4), 357-364.

16. Yokobori, S., Hosein, K., Burks, S., Sharma, I., Gajavelli, S., \& Bullock, R. (2013). Biomarkers for the clinical differential diagnosis in traumatic brain injury-a systematic review. CNS neuroscience \& therapeutics, 19(8), 556-565.

17. Cheung, Y. B., Yeo, K. K., Chong, K. J., Khoo, E. Y. H., \& Wee, H. L. (2019). Measurement equivalence of the English, Chinese and Malay versions of the World Health Organization quality of life (WHOQOL-BREF) questionnaires. Health and quality of life outcomes, 17(1), 1-6.

18. Wenqian Z., Jiapeng L., Yifei S., Jiange $H$. (2016). Changes in postoperative cognitive function during off-pump coronary artery bypass graft surgery: dose-response of propofol. Int. J. Clin. Exp. Med, V. 9, N6. P. 10939-10946. 
19. Liu, W. J., Musa, R., Chew, T. F., Lim, C. T., Morad, Z., \& Bujang, A. (2014). Quality of life in dialysis: A Malaysian perspective. Hemodialysis International, 18(2), 495-506.

20. Cheung, Y. B., Yeo, K. K., Chong, K. J., Khoo, E. Y. H., \& Wee, H. L. (2019). Measurement equivalence of the English, Chinese and Malay versions of the World Health Organization quality of life (WHOQOL-BREF) questionnaires. Health and quality of life outcomes, 17(1), 1-6. 